\title{
Effects of Joint Clearance on the Motion Accuracy of Robotic Manipulators
}

\author{
Selçuk Erkaya* \\ Erciyes University, Engineering Faculty, Mechatronics Engineering Department, Turkey
}

Accuracy is one of the key features that improves the quality of robotic manipulators for many industrial and medical applications. Even with an accurate design and manufacturing process, clearance problems in joints cannot be eliminated in articulated systems. This leads to a loss of accuracy in robotic manipulation. In this study, the effects of joint clearance in a robotic system are investigated. The need for the trajectory of end effector is considered to analyse the motion sensitivity. The kinematics and dynamics of a six-DOF robot with joint clearance are evaluated relative to a robot without joint clearance. Different scenarios for clearance values and working periods are performed to fulfil the required motion task. A neural model is also used to predict the trajectory deviations during the working process. The results show that clearance has a significant role in the motion sensitivity of robotic manipulations. Trajectory errors can also be determined by using a dynamic neural predictor with suitable input variables.

Keywords: Trajectory error, joint clearance, NARX model, 3D motion accuracy, uncertainty

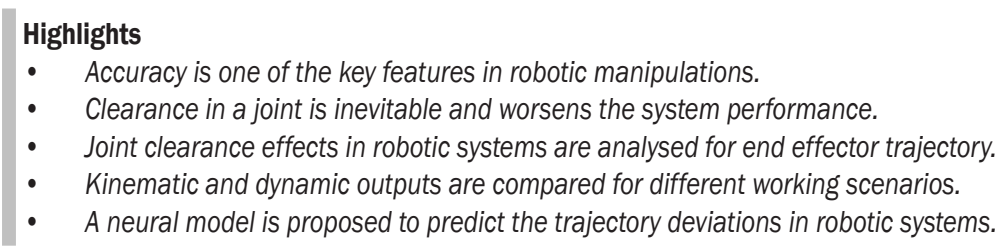

\section{INTRODUCTION}

Robots have been introduced in many industrial and medical areas where high accuracy, repeatability, and operation stability are desired. These are key features for robots. Some error sources in robots originate from assemblage, servo actuator resolution, reducer backlash, and joint clearances [1]. These errors reduce the accuracy of the robot and must be controlled to ensure the quality of the desired movement. In this regard, increased importance has been given to the accuracy of robots through various contributions in the relevant literature [2] to [5]. In comparison to machine tools, industrial robots are flexible and relatively cheaper in terms of cost. At the same time, such robots are susceptible to errors from many sources due to their serial structure. To ensure the positioning accuracy of a robot end effector as well as to reduce the manufacturing cost of the robot, it is necessary to quantify the influence of the uncertain factors and optimally allocate the tolerances.

A novel and simple approach to identify the positional and directional errors due to the joint clearance of linkages and manipulators based on a geometrical model was introduced [6]. A general probability density function of the endpoint of planar robots with joint clearance was established to derive the distribution functions for any position tolerance zone and any joint distribution type [7]. Some errors arising from link stiffness and clearances were considered to predict the accuracy of the parallel devices [8]. By considering the positional and directional errors of the robot hand and the manufacturing cost, the optimal allocation of joint tolerances was investigated. Interval analysis was used to predict the errors in the manipulator performance [9]. Singularity analysis and modelling of the joint clearance effects on the parallel robot's accuracy were conducted. An analytical model was presented to easily predict the pose error for a given external load, a nominal pose and the structural parameters [3]. A procedure to calculate the positional error in parallel manipulators due to both clearances and elastic deformations was proposed [10]. For analysing the location of the discontinuities, a methodology was presented and the advantages of approach were analysed using a $5 \mathrm{R}$ planar mechanism [11]. The effect of joint flexibility on the dynamic performance of a serial spatial robot arm with rigid links was studied by using three developed models [12]. A novel method based on trajectory planning to avoid detachment of the joint elements of a manipulator with clearances was presented. An improved detachment criterion for the different joint types was proposed [13]. The clearance effects on an industrial robot were studied during 2D welding operations. The kinematics and 
dynamics of robots were investigated for different clearance sizes [14]. The kinematic sensitivity of a robotic system with joint clearances was studied and tested for the effectiveness of the proposed model [15]. A methodology to analyse the assembly conditions and compute the maximum pose errors of parallel manipulators was presented by considering geometric errors, joint clearances, link flexibility, and joint elasticity [1]. A space robot manipulator system was considered to analyse the joint clearance effects. A computational methodology based on the nonlinear equivalent spring-damper and Coulomb friction models was proposed [16]. The effect of joint error on the positional accuracy of a robotic manipulator was presented. A serial chain two-revolute joint planar manipulator was modelled. Under the influence of the joint clearance, a formulation was presented to analyse the positional accuracy of the end effector [17]. A spherical parallel manipulator with clearance and manufacturing error was analysed to determine the pose error of the platform in the presence of these imperfections [18]. The trajectory of a walking mechanism in a mobile robot with joint clearance was studied. A neural-fuzzy model and genetic algorithm approaches were designed to improve the system performance [19]. The effects of joint clearance, link flexibility and lubrication on the kinematics and dynamics of mechanisms were extensively performed with analytical and numerical studies [20] to [22]. For improving the mechanism precision, optimization methods were also introduced to decrease the deviations owing to the clearance joint [23] to [25]. Artificial neural networks were used to evaluate the vibration characteristics of a mechanism with or without joint clearance [26] and [27]. Both theoretical and experimental studies about joint clearance were presented [28]. The effects of joint clearances on the kinematics and dynamics of planar and spatial mechanisms with rigid and elastic links were also investigated [29] to [35]. Dry contact including the friction and lubrication effects between journal and bearing parts, different sizes of clearances and joint types were investigated in many case studies. A general and comprehensive approach was proposed to automatically adjust the time step to simplify and increase the computational ability in multibody systems. 2D and 3D partly compliant mechanisms having joint clearance were studied to show the positive effects of flexural pivot on the undesired effects of joint clearance [36] and [37]. A general computer-aided model of a 3D revolute clearance joint in multibody dynamic solvers was presented [38]. A new technique for assessing the influence that the clearance of spatial revolute joints has on the kinematics and dynamics of multibody systems was presented [39]. Examination and comparison of several friction force models dealing with different friction phenomena in the context of multibody system dynamics were presented [40] and [41]. A comparative study on the most relevant existing viscoelastic contact force models was studied [42]. A critical review was presented about the existing knowledge on the computational model of normal directional impact on rigid bodies [43].

It is clear that even with an accurate design and manufacturing process for the whole system, it is not completely possible to eliminate the clearance problem in joints. In this study, motion insensitivities arising mainly from joint clearance on a robot manipulator, which can be used for laser cutting, welding, medical applications, etc., were considered. Both the trajectory of the end effector as a kinematic characteristic and necessary force/torque as a dynamic characteristic were investigated. A dynamic neural model is proposed to predict the trajectory deviations arising from joint clearance. It is possible to evaluate the end effector deviations from the desired trajectory. The outputs of this study can be used to obtain the necessary control outputs for improving the motion sensitivity by a robust controller design. This paper is organized as follows. The mathematical model of the clearance joint and motion equation of the robot manipulator are outlined in Section 1. The basic theory of the neural predictor is given in Section 2. The obtained results and conclusions are summarized in Sections 3 and 4, respectively.

\section{MODELLING OF JOINT CLEARANCE, CONTACT FORCE, AND MOTION EQUATION}

Clearance can be considered to be an imperfect joint characteristic. It is inevitable, due primarily to manufacturing errors, assemblage, and wear. In fact, a suitable value of clearance in the joint parts is essential to allow the relative motion of the adjacent links. In the presence of joint clearance, different motion types between the joint parts can be observed, that is, free-flight, impact, and continuous contact modes. These motion types fully affect the kinematic and dynamic performances of the systems. During the current trajectory, it is seen that the clearance joint exhibits nearly a similar characteristic of a 2D planar revolute joint with clearance. Due to computational efficiency, neural predictor characteristics and the robust controller design for the next studies, this negligible 3D effect of this joint is not considered. As 
given in the literature [23] and [29], radial clearance in a joint can be defined as the difference between the journal and bearing radii (Fig. 1).

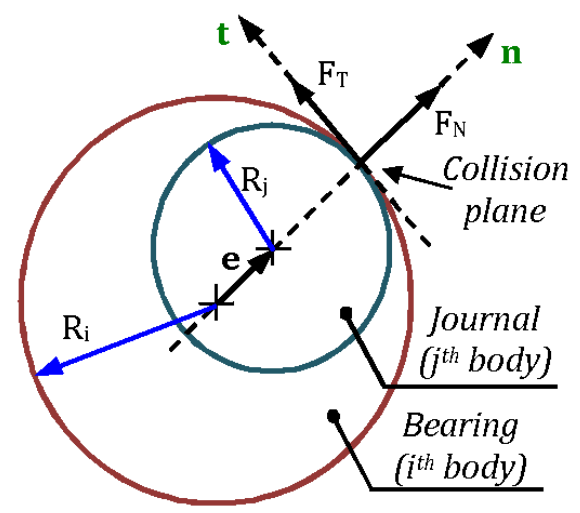

Fig. 1. Contact forces at the collision plane

The bearing and journal are parts of the $i^{\text {th }}$ and $j^{\text {th }}$ bodies, respectively. The relative penetration depth $(\delta)$ between the journal and bearing is outlined as [29] and [34]:

$$
\delta=e-\mathrm{c}
$$

in which $e$ is the magnitude of the clearance vector between the bearing and journal centres, and $\mathrm{c}$ is the radial clearance. In a clearance joint, the force is explained by two different situations. The first one is no contact forces $\left(\mathbf{F}_{\mathbf{C}}\right)$ if the joint parts are not in contact. Otherwise, there is a contact between joint parts, and the contact-impact forces are modelled according to a nonlinear dissipative force model based on the Hertzian contact theory (normal force, $\mathrm{F}_{\mathrm{N}}$ ) and Coulomb's friction law (tangential force, $\mathbf{F}_{\mathbf{T}}$ ). These two conditions can be given as [29]:

$$
\begin{array}{lll}
\mathbf{F}_{\mathbf{C}}=0 & \text { if } & \delta<0, \\
\mathbf{F}_{\mathbf{C}}=\mathrm{F}_{\mathrm{N}}+\mathbf{F}_{\mathrm{T}} & \text { if } & \delta \geq 0,
\end{array}
$$

when the magnitude of the clearance vector is greater than radial clearance, an impact occurs, and the penetration depth is calculated using Eq. (1). The contact force is modelled as a spring-damper element. If this element is linear, the approach is known as the Kelvin-Voigt model. When the relation is nonlinear, the model is generally based on the Hertz contact law [29] and [44]. In the case of an unlubricated joint, the Hertzian contact force model is an appropriate choice [44]. While the original Hertzian model does not include any energy dissipation, an extension by Lankarani and Nikravesh includes energy loss due to internal damping. The contact force model is key to describing the collision dynamics between the journal and bearing in a clearance joint [45] and [46]. Due primarily to the simplicity of its contact force model, applicability to impact in multibody systems, easy calculation, and fast convergence (inclusion of energy dissipation modelling upon impact), the model developed by Lankarani and Nikravesh [47] is widely used in the dynamics of multibody systems with joint clearance. The normal force is expressed as [29]:

$$
\mathrm{F}_{\mathrm{N}}=\mathrm{K} \delta^{(3 / 2)}+\mathrm{D} \dot{\delta},
$$

where the first term represents the elastic force component and the second term explains the energy dissipation. $\mathrm{K}$ is the generalized stiffness parameter and $\mathrm{D}$ is the hysteresis damping coefficient. $\mathrm{K}$ depends on the geometry and physical properties of the contacting surfaces and is defined by [29]:

$$
\mathrm{K}=\frac{4\left(\mathrm{E}_{\mathrm{i}} \mathrm{E}_{\mathrm{j}}\right)}{3\left(\mathrm{E}_{\mathrm{j}}\left(1-v_{\mathrm{i}}^{2}\right)+\mathrm{E}_{\mathrm{i}}\left(1-v_{\mathrm{j}}^{2}\right)\right)}\left(\frac{\mathrm{R}_{\mathrm{i}} \mathrm{R}_{\mathrm{j}}}{\mathrm{R}_{\mathrm{i}}-\mathrm{R}_{\mathrm{j}}}\right)^{1 / 2},
$$

$v$ and E are Poisson's coefficient and Young's modulus associated with journal and bearing parts. The hysteresis damping coefficient is outlined as [29]:

$$
\mathrm{D}=\left[3\left(1-\zeta^{2}\right) \mathrm{K} \delta^{3 / 2}\right] / 4 v_{0},
$$

where $\zeta$ is the restitution coefficient, and $v_{0}$ is the initial impact velocity. Friction is a complex phenomenon that comprises the interaction between the surfaces of contacting bodies and may lead to different friction regimes, such as sliding and sticking. Generally, Coulomb's friction model is used to represent the friction response in impact and contact process. However, the definition of Coulomb's friction law poses numerical difficulties when the relative tangential velocity is near zero. In the current study, a modified Coulomb friction model is used to represent the friction behaviour between the journal and bearing [29] and [48].

$$
\mathbf{F}_{\mathbf{T}}=-\mu\left(v_{T}\right) \mathrm{F}_{\mathrm{N}}\left(\mathrm{v}_{\mathrm{T}} /\left|\mathrm{v}_{\mathrm{T}}\right|\right),
$$

where $\mu\left(v_{T}\right)$ is the coefficient of friction. It is a function of relative sliding velocity $\left(v_{T}\right)$ in the collision point of journal and bearing, which is the velocity component in the tangential direction $\mu\left(v_{T}\right)$, which is not a constant, is introduced in the modified Coulomb friction model. $\mu\left(v_{T}\right)$ is a function of the tangential sliding velocity, which can represent the friction behaviour in impact and contact process as well as the viscous and microslip phenomenon in relative low-velocity cases more accurately. Furthermore, the modified Coulomb friction model can avoid the case of abrupt change of friction in the numerical calculation as the change 


$$
\mu\left(v_{T}\right)= \begin{cases}-\mu_{\mathrm{d}} \operatorname{sign}\left(v_{t}\right) & \text { for }\left|v_{t}\right|>v_{d} \\ -\left\{\mu_{\mathrm{d}}+\left(\mu_{\mathrm{s}}-\mu_{\mathrm{d}}\right)\left(\frac{\left|v_{t}\right|-v_{s}}{v_{d}-v_{s}}\right)^{2}\left[3-2\left(\frac{\left|v_{t}\right|-v_{s}}{v_{d}-v_{s}}\right)\right]\right\} \operatorname{sign}\left(v_{t}\right) & \text { for } v_{s} \leq\left|v_{t}\right| \leq v_{d}, \\ \mu_{\mathrm{s}}-2 \mu_{\mathrm{s}}\left(\frac{v_{t}+v_{s}}{2 v_{s}}\right)^{2}\left(3-\frac{v_{t}+v_{s}}{v_{s}}\right) & \text { for }\left|v_{t}\right|<v_{s}\end{cases}
$$

of velocity direction [29] and [48]. $\mu\left(v_{T}\right)$ is defined as Eq. (7), where $\mu_{\mathrm{s}}$ and $\mu_{\mathrm{d}}$ are the static and dynamic friction coefficients, respectively. $v_{s}$ and $v_{d}$ denote the critical velocities of the static and maximum dynamic frictions, respectively. Fig. 2 shows how the dynamic friction coefficient varies with slip velocity [41] and [48].

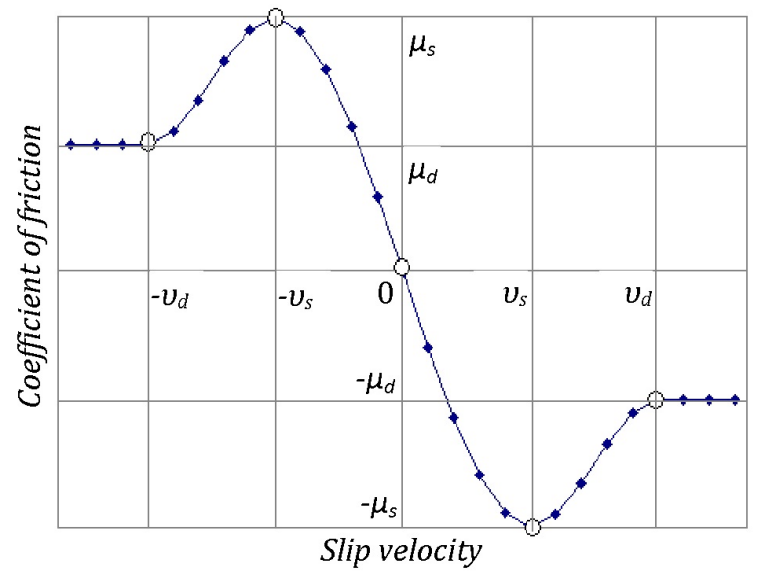

Fig. 2. Friction coefficient and slip velocity

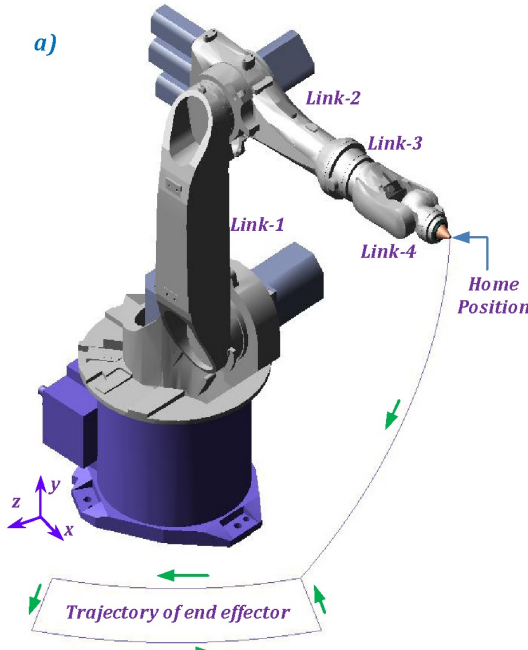

A six-axis industrial robot was considered for the theoretical analysis. This robot is mainly used for laser cutting, welding, handling, assembly etc. The robot manipulator has six degrees of freedom when all joints are perfect, as shown in Fig. 3. Different sizes of artificial clearance and working period were used to investigate the effects of joint clearance on the motion accuracy of the robot manipulator. Joint clearance leads to different motion modes such as free-flight, impact and continuous contact. Therefore, a dynamic model of the robot manipulator considering joint clearance must be developed. In case of free-flight mode, the dynamic equation for the robot manipulator is given as follows,

$$
\begin{aligned}
\mathbf{M}(q(t)) \ddot{\mathbf{q}}(\mathrm{t})+\mathbf{C}(q(t), \dot{q}(t)) \dot{\mathbf{q}}(\mathrm{t})+\ldots \\
\ldots+\mathbf{Q}(\dot{q}(t))+\mathbf{G}(q(t))=\mathbf{F}(\mathrm{t}),
\end{aligned}
$$

where $\mathbf{q}$ is the generalized coordinate column matrix. $\mathbf{M}(q(t))$ denotes the inertia matrix of the robot manipulator. $\mathbf{C}(q(t), \dot{q}(t))$ is the vector of centrifugal and coriolis terms. $\mathbf{Q}(\dot{q}(t))$ represents the friction term, which consists of the viscous and dynamic

Fig. 3. Robot manipulator: a) trajectory of end effector, b) motion capability 
frictions. $\mathbf{G}(q(t))$ and $\mathbf{F}(t)$ denote the vector of gravity term and the generalized force matrix, respectively.

In case of impact and continuous contact modes, contact force takes places between the journal and bearing in the joint. Therefore, the dynamic equation is outlined as:

$$
\begin{array}{r}
\mathbf{M}(q(t)) \ddot{\mathbf{q}}(\mathrm{t})+\mathbf{C}(q(t), \dot{q}(t)) \dot{\mathbf{q}}(\mathrm{t})+\mathbf{Q}(\dot{q}(t))+\ldots \\
\ldots+\mathbf{G}(q(t))=\mathbf{F}(\mathrm{t})+\mathbf{F}_{\mathbf{C}}(\delta, \dot{\delta}),
\end{array}
$$

where $\mathbf{F}_{\mathbf{c}}(\delta, \dot{\delta})$ is the contact force that comprises the normal and tangential components. One of the most important and complex parts of the simulation of multibody systems comprising the contact-impact analysis procedure is the detection of the precise instant of impact. In addition, the model used to characterize the contact between the bodies requires the knowledge of the pre-impact conditions, that is, the impact velocity and the direction of the normal to the colliding surface. Neither the contact duration nor the penetration can be predicted from the pre-impact conditions due to the influence of the kinematic constraints and other interactions on the bodies of the complete system [35]. The characteristics of the robot manipulator are outlined in Table 1. The dynamic simulation parameters of the robot manipulator with joint clearance are outlined in Table 2 .

Table 1. Characteristics of robot manipulator

\begin{tabular}{ccccccccc}
\hline \multirow{2}{*}{$\begin{array}{l}\text { Robot } \\
\text { Links }\end{array}$} & $\begin{array}{c}\text { Length } \\
{[\mathrm{mm}]}\end{array}$ & $\begin{array}{c}\text { Mass } \\
{[\mathrm{kg}]}\end{array}$ & \multicolumn{5}{c}{ Mass moment of inertia characteristics [kgm²] } \\
\cline { 5 - 9 } & & & $J_{x x}$ & $J_{y y}$ & $J_{z z}$ & $J_{x y}$ & $J_{y z}$ & $J_{z x}$ \\
\hline Link 1 & 680 & 74.08 & 3.26 & 0.32 & 3.25 & $1.8410^{-3}$ & 0.145 & $-1.32 \times 10^{-3}$ \\
\hline Link 2 & 402 & 43.51 & 0.336 & 1.407 & 1.362 & $-5.28 \times 10^{-3}$ & $-7.46 \times 10^{-3}$ & $9.78 \times 10^{-3}$ \\
\hline Link 3 & 268 & 7.87 & $1.83 \times 10^{-2}$ & $6.46 \times 10^{-2}$ & $5.89 \times 10^{-2}$ & $1.83 \times 10^{-5}$ & $4.02 \times 10^{-6}$ & $3.68 \times 10^{-3}$ \\
\hline Link 4 & 115 & 2.22 & $2.09 \times 10^{-3}$ & $4.9 \times 10^{-3}$ & $4.86 \times 10^{-3}$ & $7.61 \times 10^{-10}$ & $1.13 \times 10^{-9}$ & $5.56 \times 10^{-4}$ \\
\hline
\end{tabular}

Table 2. Simulation parameters of robot manipulator

\begin{tabular}{lccc}
\hline Description & Value & Description & Value \\
\hline Dynamic friction coefficient & 0.01 & Young's modulus & $71.7 \mathrm{GPa}$ \\
\hline Restitution coefficient & 0.9 & Integration step size & $1 \times 10^{-3} \mathrm{~s}$ \\
\hline Poisson's coefficient & 0.33 & Integration tolerance & $1 \times 10^{-5}$ \\
\hline
\end{tabular}

\section{NONLINEAR AUTOGRESSIVE EXOGENOUS (NARX) MODEL}

Neural network applications have rapidly expanded over the last two decades owing to the advances in computer science and sensor technologies [49]. In case of system identifications using neural networks, the main purpose is usually to define a dynamically valid model that can be used for system analysis [26]. The NARX is used for modelling nonlinear dynamical systems [50]. It is one of the artificial neural network models for time series prediction. It learns the behaviour of a system in a more effective way than other neural networks do (i.e., the learning gradient algorithm is better in NARX) and converges much faster and generalizes better than other networks [51] and [52]. It has been demonstrated that it can predict the behaviours of nonlinear dynamics systems and is particularly useful for time series modelling [53] and [54]. The structure of the NARX model is similar to the traditional multi-layered perceptron (MLP) model. Among the various Artificial Neural Network (ANN) models, the MLP model is one of the most widely used for prediction due primarily to its simple and flexible nature. Both the MLP and the NARX models consist of an input, a hidden layer, and an output layer. In addition, the NARX model feeds the time history of the output signal to the input layer as part of the inputs. The current input signal together with its time history acts as the other part of the inputs to the model. The number of output neurons is equal to the number of output variables of the problem to be solved. The number of hidden neurons is determined by the user. Assume $\Gamma$ is the function of the NARX model. It correlates the input time series $\left\{u_{t}\right\}_{t=0}^{T}$ and the output time series $\left\{y_{t}\right\}_{t=0}^{T}$ by the Eq. (10).

$$
y_{t}=\Gamma\left(y_{t-1}, y_{t-2}, \ldots, y_{t-\tau_{y}}, u_{t}, u_{t-1}, \ldots, u_{t-\tau_{u}}\right) \text {, }
$$

where $u_{t}$ and $y_{t}$ denote the input and output of the network at time $t$, the function $\Gamma$ is a nonlinear function, and $\tau_{y}$ and $\tau_{u}$ are the time lags for the input and output series, respectively. Fig. 4 gives the general architecture of the NARX model. 


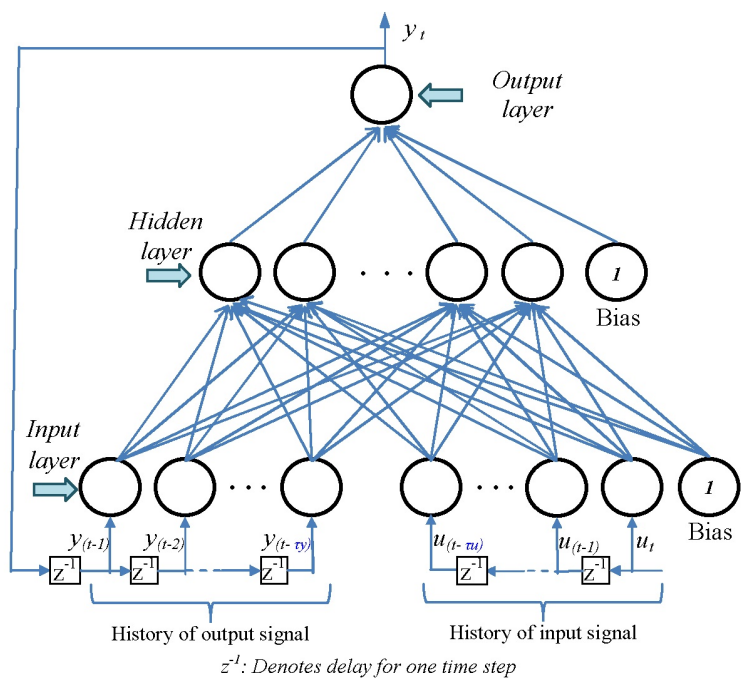

Fig. 4. Architecture of the NARX model

In this study, the deviations of the end effector are considered as the output of the NARX model. The inputs of the NARX model consist of the time histories of the necessary torques and path components, specifications of the joint with clearance (clearance value, number of joints with clearance, and the location of joint with clearance). Each neuron has a bias input. Input and output layers have the linear activation functions. The sigmoid function is also used in four hidden layers with ten neurons. In this study, the Levenberg-Marquardt algorithm is adopted for the learning process due to its fast convergence properties [55]. The performance index used in NARX model training is the mean squared error (MSE), which is one of the typical performance functions. The NARX model was trained using the Neural Network Toolbox of MATLAB [50]. For training, validation and testing purposes, the complete procedure performs the data during three steps. The training step is used to adjust NARX weights. The validation step is used for selecting the model variant. The last step is testing. It is used to evaluate the chosen model against unknown data. In this work, 1500 and 750 data are used for 12 and 6 second working periods, respectively. Of all data, $50 \%$ were used in training; $20 \%$ and $30 \%$ of all data were used for validation and testing, respectively. It is seen from the simulation; these data numbers are adequate for designing a stable neural model.

\section{RESULTS}

A theoretical study was implemented to analyse the motion accuracy of robot manipulators with joint clearance. Exaggerated clearance values as an imperfect joint characteristic were adjusted in a reference joint (joint 5) as $0.2 \mathrm{~mm}, 0.4 \mathrm{~mm}$ and 0.6 $\mathrm{mm}$ for three case studies and comparisons. The working periods were considered as 6 and 12 seconds. The robot manipulator has six degrees of freedom when all joints are ideal or perfect. By using suitable input variables, a dynamic neural network was used
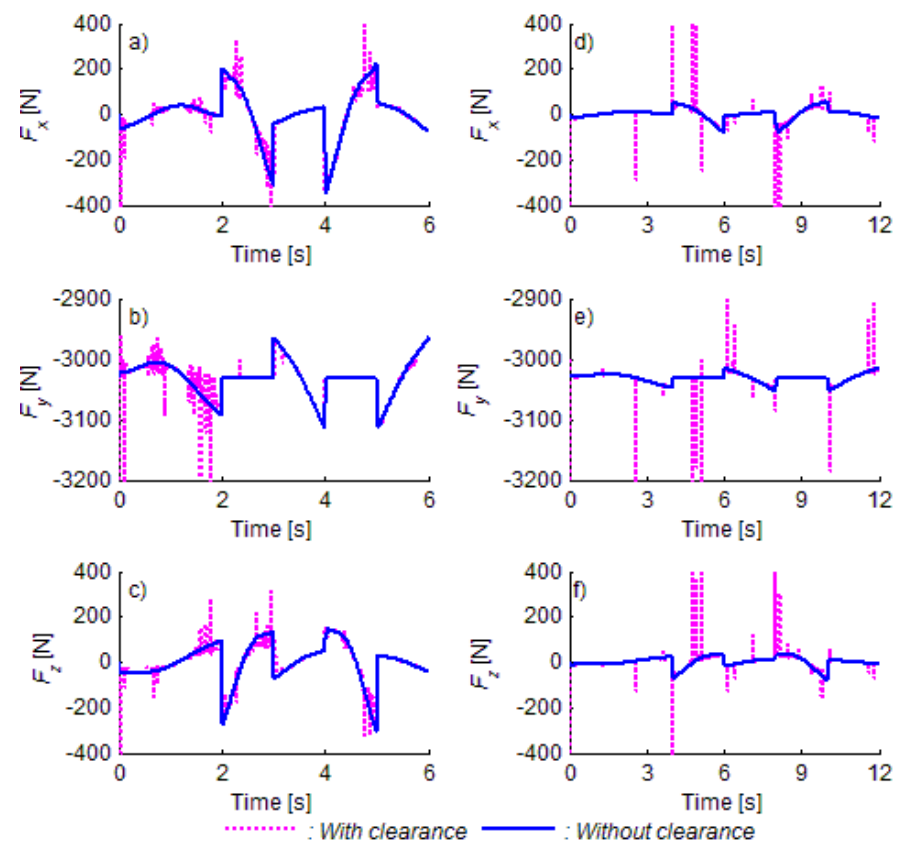

Fig. 5. Forces for $0.2 \mathrm{~mm}$ clearance: a), b) and c) working for 6 seconds, d), e) and f) working for 12 seconds 
for predicting the clearance-induced deviations in the robot trajectory. In case of perfect, imperfect joint characteristics and neural modelling, all simulations were performed using a Pentium Core 2 Quad Q6600 computer. At the beginning of the manipulator motion, it was considered that the journal centre coincides with the bearing centre. To show the clear effects of joint clearance, the force components of joint 1 are considered. Figs. 5 to 7 give the force components of joint 1 for different scenarios. The same scales are used for easy comparison and evaluation of the force components. However, the maximum values of some peaks are beyond those used scales. As shown in the figures, the robot manipulator has greater force variation in a short working period [14]. The existence of joint clearance causes impulse-type contact forces.
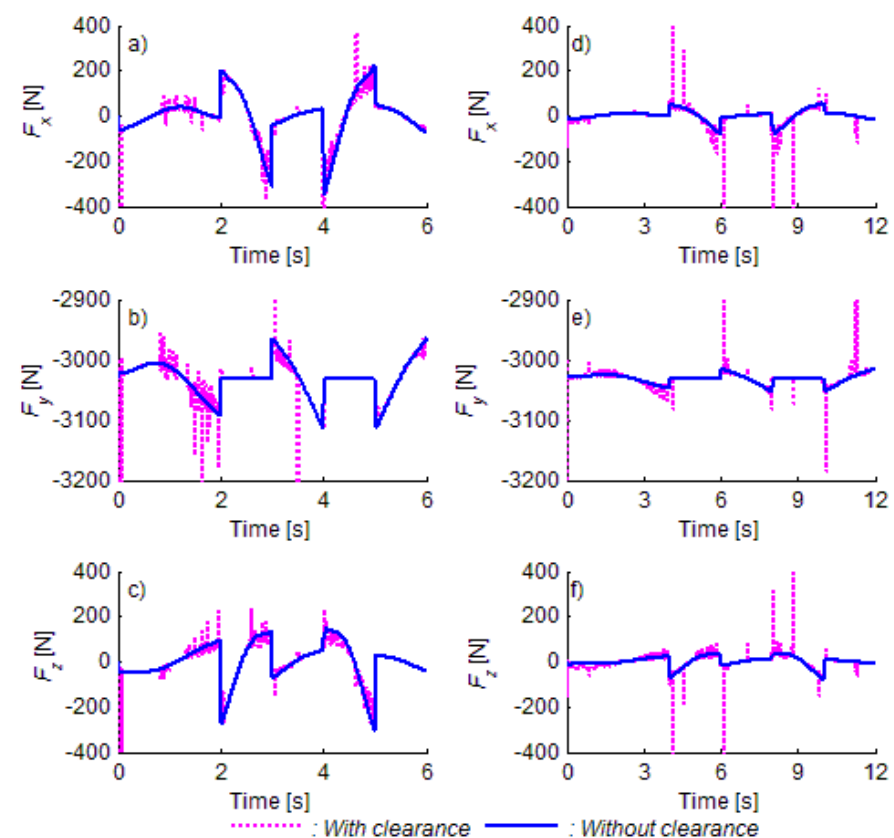

Fig. 6. Forces for $0.4 \mathrm{~mm}$ clearance: a), b) and c) working for 6 seconds, d), e) and f) working for 12 seconds
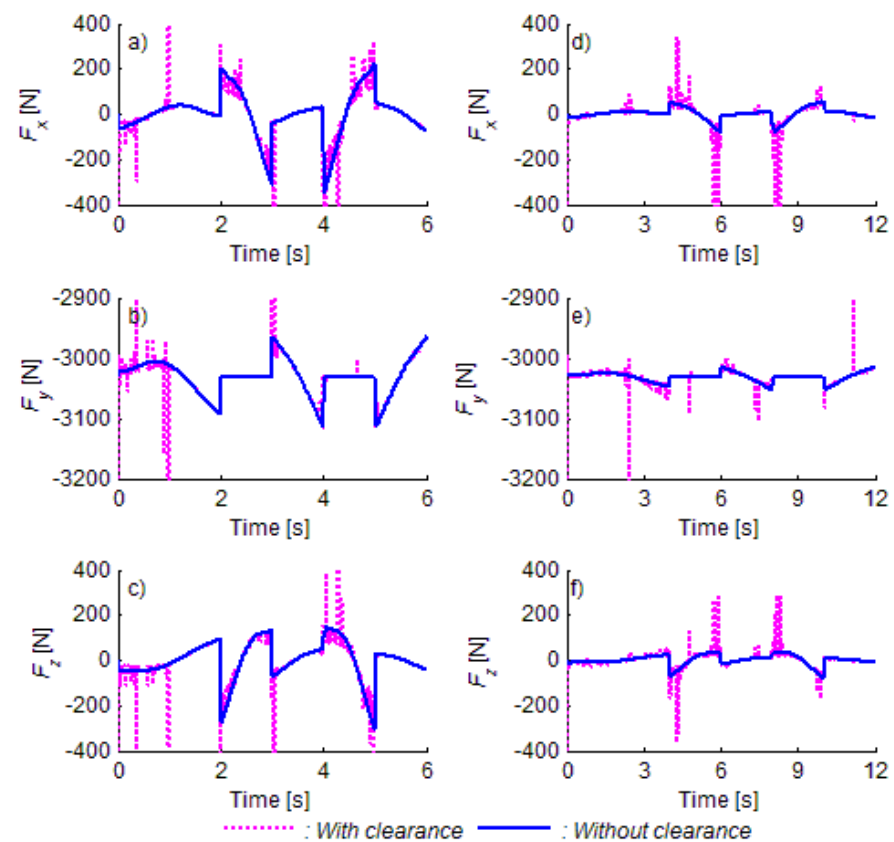

Fig. 7. Forces for $0.6 \mathrm{~mm}$ clearance: a), b) and c) working for 6 seconds, d), e) and f) working for 12 seconds 
These forces occur during a short interval [16] and [48]. Particularly, these peaks are seen at the beginning of the motion, and there is an instant change in force characteristics.

Similarly, in the short working period, the robot manipulator with joint clearance has higher peak frequency [16] and [48]. When the clearance size is increased, there is also an increase in the amplitude of the contact force [22], [36] and [37]. This force characteristic not only reduces the motion sensitivity and repeatability of the robot manipulator, but also leads to a decrease in its vibration and noise quality [20], [22] and [26]. The effects of clearance on torque characteristics are given in Figs. 8 to 10 for different scenarios of clearance value and working period.

The Cartesian components of joints 1 and 2 are used to show the clearance effects on the system torque. Similarly, when the working period for the
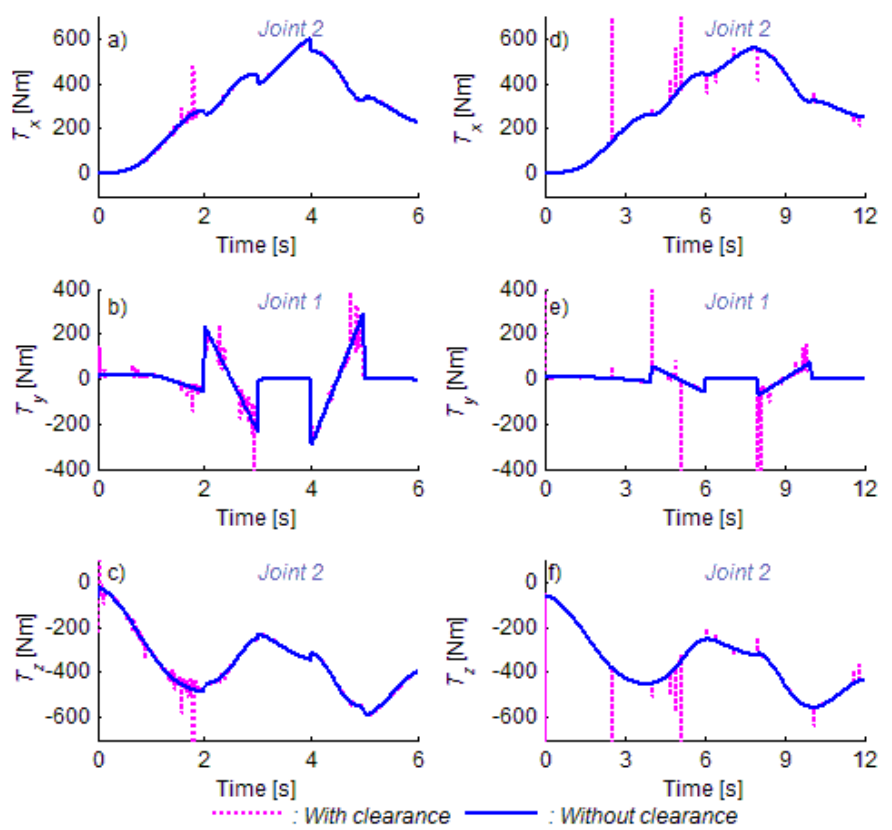

Fig. 8. Torques for $0.2 \mathrm{~mm}$ clearance: a), b) and c) working for 6 seconds, d), e) and f) working for 12 seconds
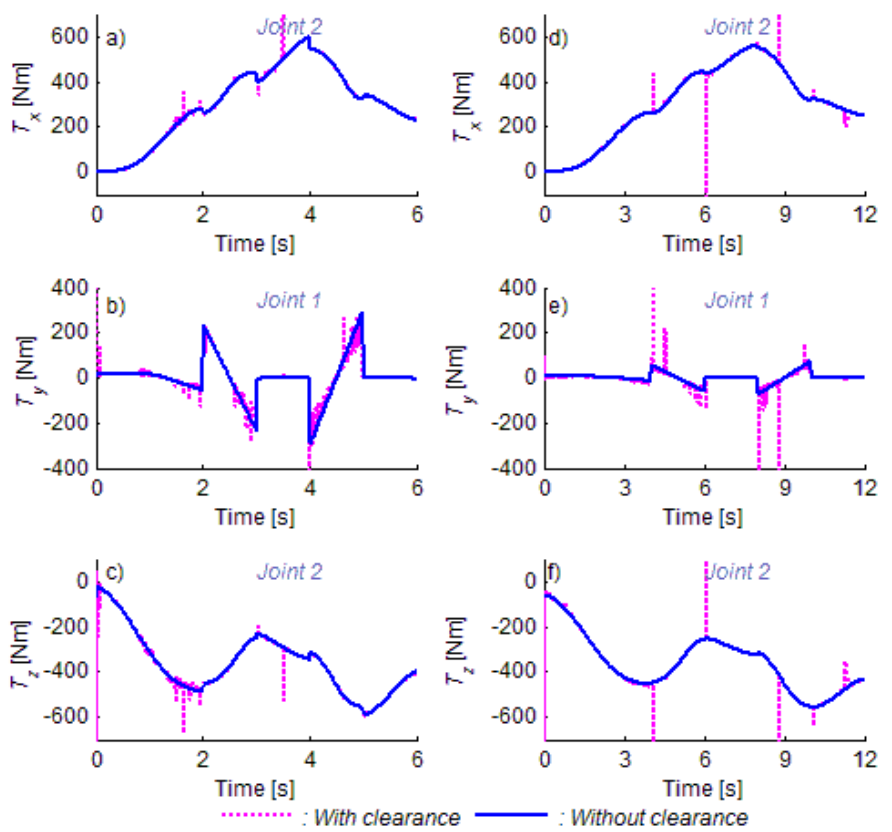

Fig. 9. Torques for $0.4 \mathrm{~mm}$ clearance: a), b) and c) working for 6 seconds, d), e) and f) working for 12 seconds 
same task is reduced, the required torque is also increased. The robot manipulator with joint clearance has some peaks on torques [20]. As seen in the working area, the y component of torque is more important for accomplishing the related trajectory. This can be seen from the peak values and peak frequency. By evaluating the force and torque components together, joint clearance naturally affects all the system dynamics [31], [33], and [34]. The impulsive behaviour arising from a joint with clearance leads to the degradation of the overall system performance [28] and [48]. Similarly, clearance size has a significant role in system response. The dynamics of the robot manipulator are obviously different for the case of
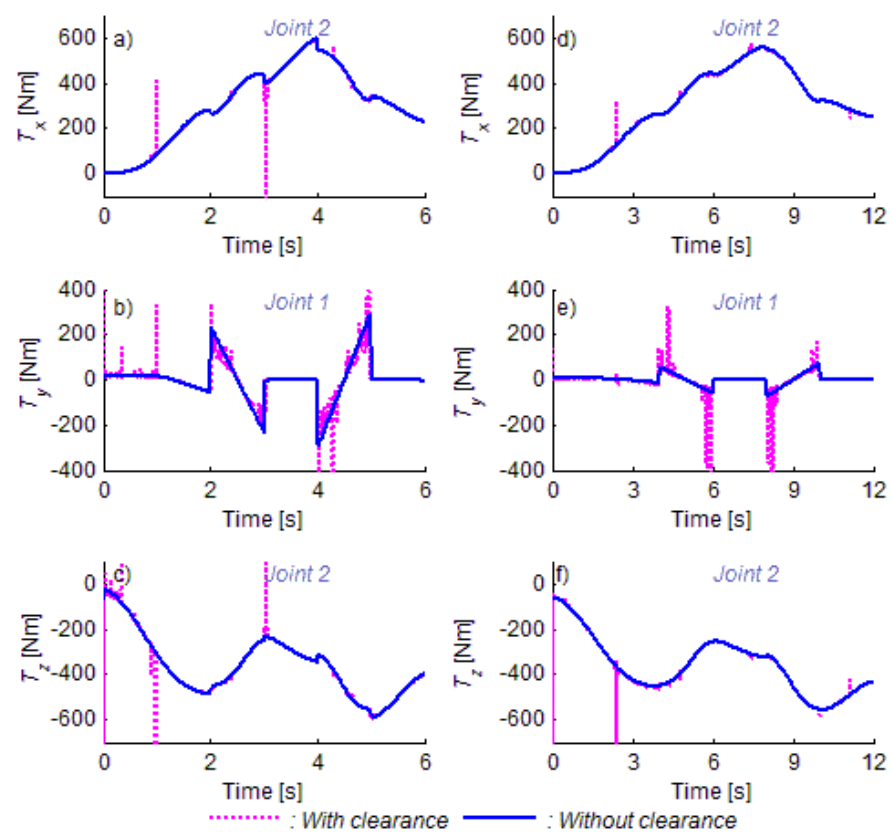

Fig. 10. Torques for $0.6 \mathrm{~mm}$ clearance: a), b) and c) working for 6 seconds, d), e) and f) working for 12 seconds
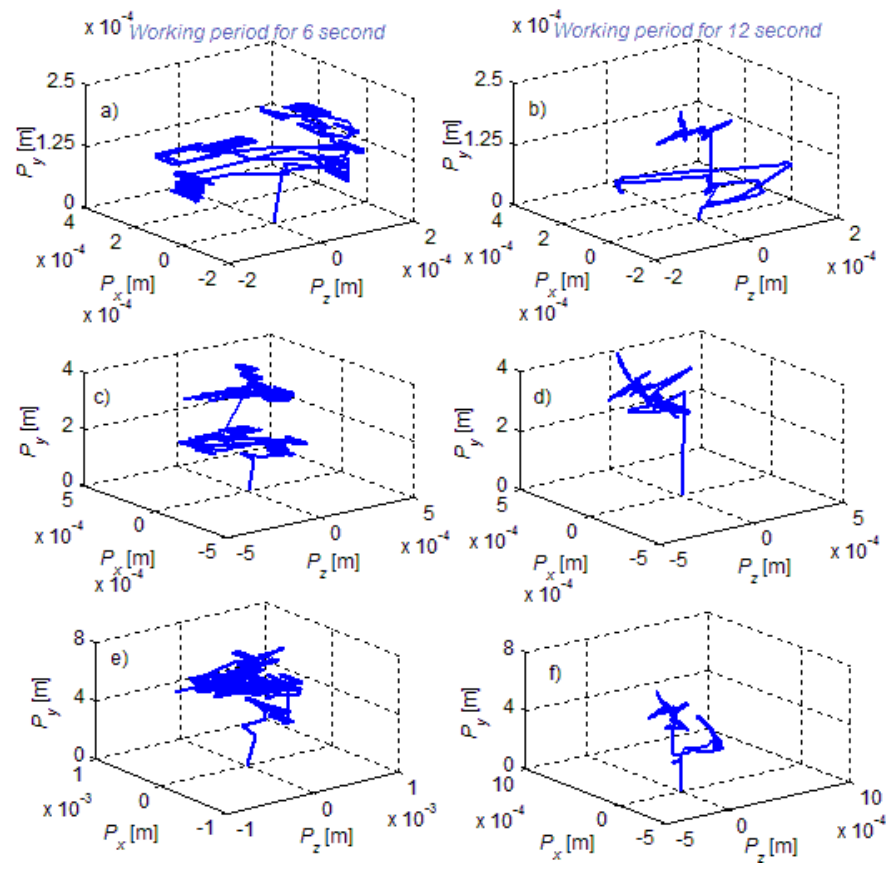

Fig. 11. Trajectory deviations for different clearance values and working periods: a) and b) $0.2 \mathrm{~mm}$ clearance value, c) and d) $0.4 \mathrm{~mm}$ clearance value, e) and f) $0.6 \mathrm{~mm}$ clearance value 
different clearance sizes and working periods. A lower clearance size leads to a small impulsive character [16]. In the case of the same task, the shorter working period is a dominant factor for peak frequency.

Fig. 11 outlines the trajectory deviations of the end effector for the case of different working periods and clearance sizes. There is a clear trajectory deviation for robot manipulators having perfect and imperfect joints [19]. Motion sensitivity and repeatability are crucial for medical and industrial robots that are used for laser cutting, high-quality welding, etc. Trajectory deviations in the robot manipulator with a clearance joint are clearly seen in Fig. 11. Increases in clearance values naturally lead to an increase in trajectory deviations [6], [14] and [23]. More chaotic behaviour in deviations is seen in the case of the shorter working period for carrying out the same task. More free-flight and then impact modes are the main reasons to fulfil the same trajectory at shorter working times at higher speeds. These deviations can be predicted by using a neural model with suitable input variables. To show the proposed network's stability for predicting the deviations in path components, a $0.6 \mathrm{~mm}$ clearance value is considered for both working conditions as a sample application (Fig. 12). As seen in the error-time figures, the NARX model can be used to predict the deviations in trajectory. The convergence capability of the neural model is very effective [24] to [27].
The obtained results are good reflections of the modelling and predicting capabilities of this model [56]. As a performance measuring criterion, the MSE is bigger in the 6-second working period than in the 12 -second working period, because the short period is the main reason for peak frequency. Similarly, more complex behaviours in the kinematics and dynamics of the robot manipulator with joint clearance are seen in this period.

Fig. 13 shows a scatter plot of the NARX model with the best fit line of the theoretical trajectory error versus the predicted one. As shown, the scatter plot of theoretical error versus NARX prediction provides a rather good insight for working periods. The correlation for the long working period is higher than for the short one. These correlations are good reflections of the proposed neural model's ability to predict the correct deviations.

\section{CONCLUSIONS}

Additional degrees of freedom originating from clearance lead to different motion modes. These are sources of uncertainties in robotic manipulations. The model manipulator has six degrees of freedom when all joints are perfect. In the current study, one joint was considered to be imperfect to show the effects of joint clearance on the system. All simulations show that much more computation time is necessary in the
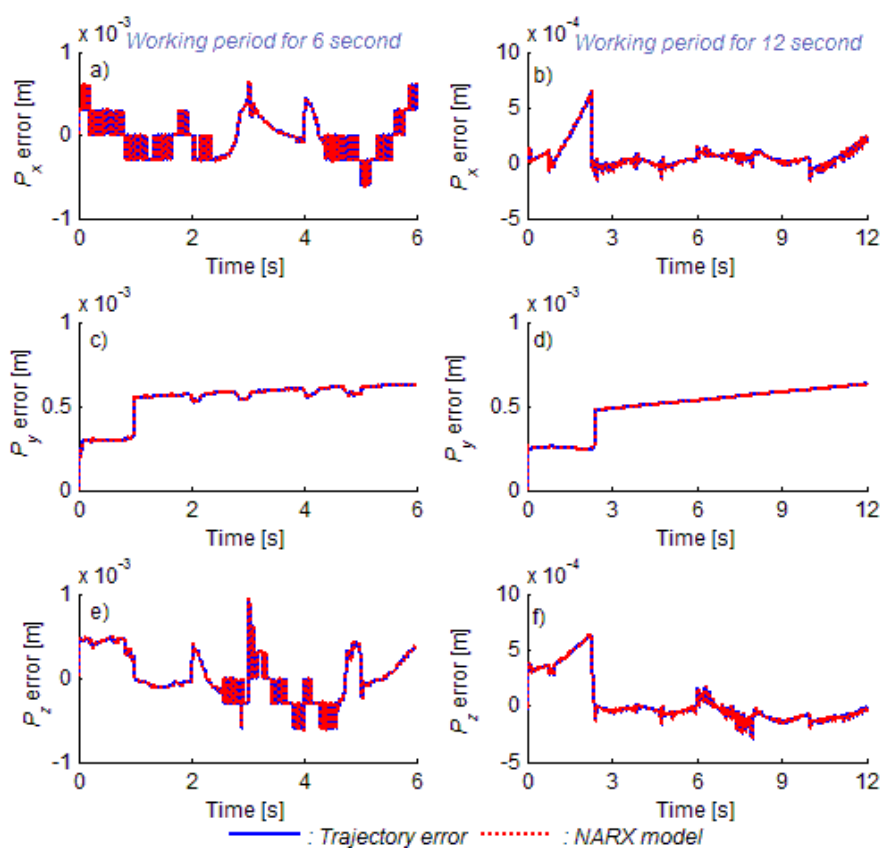

Fig. 12. Trajectory errors and NARX model for $0.6 \mathrm{~mm}$ clearance value: a) and b) $x$ component, c) and d) $y$ component, e) and f) $z$ component 

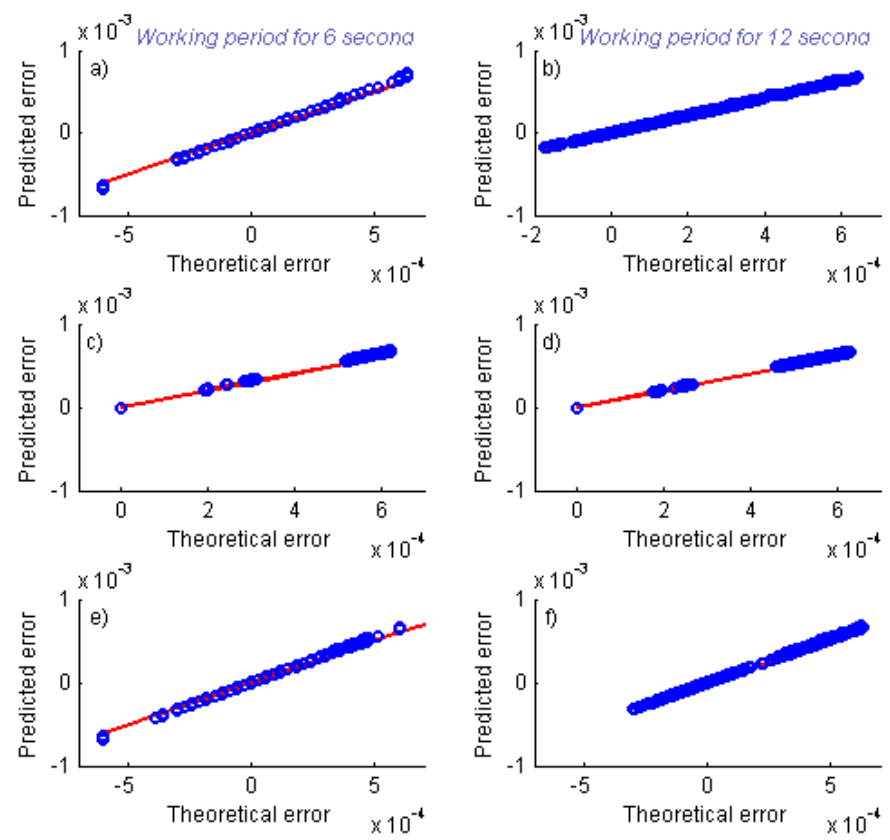

Fig. 13. Scatter plot of NARX model including a best fit line for $0.6 \mathrm{~mm}$ clearance value: a) and b) $x$ component, c) and d) y component, e) and f) z component

case of the imperfect joint approach. The existence of joint clearance has clear effects on system kinematics and dynamics, and causes impulse-type contact force/ torque during a short interval. Both clearance size and working period are important factors in peak amplitude and frequency. When the speed is increased as a result of the reduced working period for carrying out the same task, deviations in the desired trajectory are also more chaotic. An instant change in necessary force or torque leads to an impulsive effect on system outputs. Analyses of robots with joint clearance should be considered as the basis of motion accuracy in robotic manipulations even if the clearance size is small. To predict the trajectory deviations, a dynamic neural model using a suitable input matrix is proposed. This model makes the evaluation of the end effector deviations from the desired trajectory possible. By considering the working period, the approximation error of the neural predictor is smaller in the long period than it is in the short period. These outputs can be used to improve motion sensitivity by designing a robust control system for generating the necessary control outputs. For the future developments, different scenarios such as more clearance joints, lubricated joints having clearances, wear, etc. will be studied.

\section{REFERENCES}

[1] Corradi, D., Caro, S., Chablat, D., Cardou, P. (2014). Assembly conditions of parallel manipulators considering geometric errors, joint clearances, link flexibility and joint elasticity. IEEE International Conference on Robotics and Automation, $\mathrm{p}$. 4067-4072, DOI:10.1109/ICRA.2014.6907450.

[2] Al-Widyan, K., Ma, X.Q., Angeles, J. (2011). The robust design of parallel spherical robots. Mechanism and Machine Theory, vol. 46, no. 3, p. 335-343, D0l:10.1016/j. mechmachtheory.2010.11.002.

[3] Chebbi, A-H., Affi, Z., Romdhane, L. (2009). Prediction of the pose errors produced by joints clearance for a 3-UPU parallel robot. Mechanism and Machine Theory, vol. 44, no. 9, p. 1768-1783, D0I:10.1016/j.mechmachtheory.2009.03.006.

[4] Frisoli, A., Solazzi, M., Pellegrinetti, D., Bergamasco, M. (2011). A new screw theory method for the estimation of position accuracy in spatial parallel manipulators with revolute joint clearances. Mechanism and Machine Theory, vol. 46, no. 12, p. 1929-1949, D0l:10.1016/j.mechmachtheory.2011.07.004.

[5] Wu, W., Rao, S.S. (2004). Interval approach for the modeling of tolerances and clearances in mechanism analysis. Journal of Mechanical Design, vol. 126, no. 4, p. 581-592, DOI:10.1115/1.1760775.

[6] Ting, K.L., Zhu, J., Watkins, D. (2000). The effects of joint clearance on position and orientation deviation of linkages and manipulators. Mechanism and Machine Theory, vol, 35, no. 3, p. 391-401, D0I:10.1016/S0094-114X(99)00019-1.

[7] Zhu, J., Ting, K.L. (2000). Uncertainty analysis of planar and spatial robots with joint clearances. Mechanism and Machine Theory, vol. 35, no. 9, p. 1239-1256, D0l:10.1016/S0094114X(99)00076-2. 
[8] Lim, S.R., Kang, K., Park, S., Choi, W.C., Song, J.-B., Hong, D., Shim, J.K. (2002). Error analysis of a parallel mechanism considering link stiffness and joint clearances. KSME International Journal, vol. 16, no. 6, p. 799-809, D0l:10.1007/ BF02939339.

[9] Wu, W., Rao, S.S. (2007). Uncertainty analysis and allocation of joint tolerances in robot manipulators based on interval analysis. Reliability Engineering and System Safety, vol. 92, no. 1, p. 54-64, D0l:10.1016/j.ress.2005.11.009.

[10] Aginaga, J., Altuzarra, O., Macho, E., Iriarte, X. (2013). Assessing position error due to clearances and deformations of links in parallel manipulators. Journal of Mechanical Design, vol. 135, no. 4, p. 041006, D0l:10.1115/1.4023633.

[11] Altuzarra, 0., Aginaga, J., Hernández, A., Zabalza, I. (2011). Workspace analysis of positioning discontinuities due to clearances in parallel manipulators. Mechanism and Machine Theory, vol. 46, no. 5, p. 577-592, D0l:10.1016/j. mechmachtheory.2011.01.005.

[12] Zaher, M.H., Megahed, S.M. (2015). Joints flexibility effect on the dynamic performance of robots. Robotica, vol. 33, no. 7, p. 1424-1445, D0I:10.1017/S0263574714000848.

[13] Bu, W., Liu, Z., Tan, J., Gao, S. (2010). Detachment avoidance of joint elements of a robotic manipulator with clearances based on trajectory planning. Mechanism and Machine Theory, vol. 45, no. 6, p. 925-940, D0I:10.1016/j. mechmachtheory.2010.01.006.

[14] Erkaya, S. (2012). Investigation of joint clearance effects on welding robot manipulators. Robotics and ComputerIntegrated Manufacturing, vol. 28, no. 4, p. 449-457, D0I:10.1016/j.rcim.2012.02.001.

[15] Binaud, N., Cardou, P., Caro, S., Wenger, P. (2010). The kinematic sensitivity of robotic manipulators to joint clearances. ASME International Design Engineering Technical Conferences \& Computers \& Information in Engineering Conference, Volume 2: 34th Annual Mechanisms and Robotics Conference, Parts A and B, p. 1371-1380, D0l:10.1115/ DETC2010-28635.

[16] Zhao, Y., Bai, Z.F. (2011). Dynamics analysis of space robot manipulator with joint clearance. Acta Astronautica, vol. 68, no. 7-8, p. 1147-1155, D0l:10.1016/j.actaastro.2010.10.004.

[17] Jawale, H.P., Thorat, H.T. (2013). Positional error estimation in serial link manipulator under joint clearances and backlash. Journal Mechanisms Robotics, vol. 5, no. 2, p. 021003 , DOI:10.1115/1.4023556.

[18] Chaker, A., Mlika, A., Laribi, M.A., Romdhane, L., Zeghloul, S. (2013). Clearance and manufacturing errors' effects on the accuracy of the 3-RCC Spherical Parallel Manipulator. European Journal Mechanics-A/Solids, vol. 37, p. 86-95, DOl:10.1016/j.euromechsol.2012.05.009.

[19] Erkaya, S. (2013). Trajectory optimization of a walking mechanism having revolute joints with clearance using ANFIS approach. Nonlinear Dynamics, vol. 71, no. 1-2, p. 75-91, D0I:10.1007/s11071-012-0642-5.

[20] Erkaya, S., Uzmay, i. (2009). Investigation on effect of joint clearance on dynamics of four-bar mechanism. Nonlinear Dynamics, vol. 58 , p. 179-198, D0l:10.1007/s11071-0099470-7.
[21] Zheng, E., Zhu, R., Zhu, S., Lu, X. (2016). A study on dynamics of flexible multi-link mechanism including joints with clearance and lubrication for ultra-precision presses. Nonlinear Dynamics, vol. 83, no. 1-2, p. 137-159, D0l:10.1007/s11071015-2315-7.

[22] Erkaya, S., Doğan, S. (2015). A Comparative analysis of joint clearance effects on articulated and partly compliant mechanisms. Nonlinear Dynamics, vol. 81, no. 1-2, p. 323341, D0l:10.1007/s11071-015-1994-4.

[23] Erkaya, S., Uzmay, ì. (2009). Determining link parameters using genetic algorithm in mechanisms with joint clearance. Mechanism and Machine Theory, vol. 44, no. 1, p. 222-234, D0I:10.1016/j.mechmachtheory.2008.02.002.

[24] Erkaya, S., Uzmay, i. (2009). Optimization of transmission angle for slider-crank mechanism with joint clearances. Structural and Multidisciplinary Optimization, vol. 37, no. 5, p. 493-508, D0I:10.1007/s00158-008-0243-6.

[25] Erkaya, S., Uzmay, İ. (2008). A neural-genetic (NN-GA) approach for optimising mechanisms having joints with clearance. Multibody System Dynamics, vol. 20, no. 1, p. 6983, D0I:10.1007/s11044-008-9106-6.

[26] Erkaya, S. (2012). Prediction of vibration characteristics of a planar mechanism having imperfect joints using neural network. Journal of Mechanical Science and Technology, vol. 26, no. 5, p. 1419-1430, D0l:10.1007/s12206-012-0308-8.

[27] Eski, İ., Erkaya, S., Savaş, S., Yildirim, Ş., (2011). Fault detection on robot manipulators using artificial neural networks. Robotics and Computer-Integrated Manufacturing, vol. 27, no. 1, p. 115-123, D0l:10.1016/j.rcim.2010.06.017.

[28] Erkaya, S., Uzmay, i. (2010). Experimental investigation of joint clearance effects on the dynamics of a slider-crank mechanism. Multibody System Dynamics, vol. 24, no. 1, p. 81102, D0l:10.1007/s11044-010-9192-0.

[29] Flores, P., Ambrósio, J., Claro, H.C.P., Lankarani, H.M., Koshy, C.S. (2006). A study on dynamics of mechanical systems including joints with clearance and lubrication. Mechanism and Machine Theory, vol. 41, no. 3, p. 247-261, Dol:10.1016/j. mechmachtheory.2005.10.002.

[30] Flores, P. (2009). Modeling and simulation of wear in revolute clearance joints in multibody systems. Mechanism and Machine Theory, vol. 44, no. 6, p. 1211-1222, Dol:10.1016/j. mechmachtheory.2008.08.003.

[31] Erkaya, S., Uzmay, I. (2014). Modeling and simulation of joint clearance effects on mechanisms having rigid and flexible links. Journal of Mechanical Science and Technology, vol. 28, no. 8, p. 2979-2986, D0l:10.1007/s12206-014-0705-2.

[32] Tian, Q., Liu, C., Machado, M., Flores, P. (2011). A new model for dry and lubricated cylindrical joints with clearance in spatial flexible multibody systems. Nonlinear Dynamics, vol. 64, no. 1-2, p. 25-47, D0l:10.1007/s11071-010-9843-y.

[33] Erkaya, S., Uzmay, I. (2012). Effects of balancing and link flexibility on dynamics of a planar mechanism having joint clearance. Scientia Iranica, vol. 19, no. 3, p. 483-490, Dol:10.1016/j.scient.2012.04.011.

[34] Flores, P., Koshy, C.S., Lankarani, H.M., Ambrósio, J., Claro, J.C.P. (2011). Numerical and experimental investigation on multibody systems with revolute clearance joints. Nonlinear 
Dynamics, vol. 65, no. 4, p. 383-398, Dol:10.1007/s11071010-9899-8.

[35] Flores, P., Ambrósio, J. (2010). On the contact detection for contact-impact analysis in multibody systems. Multibody System Dynamics, vol. 24, no. 1, p. 103-122, D0l:10.1007/ s11044-010-9209-8.

[36] Erkaya, S., Doğan, S., Şefkatlioğlu, E. (2016). Analysis of the joint clearance effects on a compliant spatial mechanism. Mechanism and Machine Theory, vol. 104, p. 255-273, D0I:10.1016/j.mechmachtheory.2016.06.009.

[37] Erkaya, S., Doğan, S., Ulus, Ş., (2015). Effects of joint clearance on the dynamics of a partly compliant mechanism: Numerical and experimental studies. Mechanism and Machine Theory, vol. 88, p. 125-140, D0l:10.1016/j. mechmachtheory.2015.02.007.

[38] Brutti, C., Coglitore, G., Valentini, P.P. (2011). Modeling 3D revolute joint with clearance and contact stiffness. Nonlinear Dynamics, vol. 66, no. 4, p. 531-548, D0l:10.1007/s11071010-9931-z.

[39] Flores, P., Ambrósio, J., Claro, J.C.P., Lankarani, H.M. (2006). Spatial revolute joints with clearance for dynamic analysis of multibody systems. Proceedings of the Institution of Mechanical Engineers, Part-K Journal of Multibody Dynamics, vol. 220, no. 4, p. 257-271, D0I:10.1243/1464419JMBD70.

[40] Marques, F., Flores, P., Claro, J.C.P., Lankarani, H.M. (2016). A survey and comparison of several friction force models for dynamic analysis of multibody mechanical systems. Nonlinear Dynamics, vol. 86, no. 3, p. 1407-1443, D0l:10.1007/s11071016-2999-3.

[41] Pennestrì, E., Rossi, V., Salvini, P., Valentini, P.P. (2016). Review and comparison of dry friction force models. Nonlinear Dynamics, vol. 83, no. 4, p. 1785-1801, D0l:10.1007/s11071015-2485-3.

[42] Alves, J., Peixinho, N., da Silva, M.T., Flores, P., Lankarani, H.M. (2015). A comparative study on the viscoelastic constitutive laws for frictionless contact interfaces in multibody dynamics. Mechanism and Machine Theory, vol. 85, p. 172-188, D0I:10.1016/j.mechmachtheory.2014.11.020.

[43] Banerjee, A., Chanda, A., Das, R. (2017). Historical origin and recent development on normal directional impact models for rigid body contact simulation: A critical review. Archives of Computational Methods in Engineering, vol. 24, no. 2, p. 397422, Dol:10.1007/s11831-016-9164-5.

[44] Ravn, P. (1998). A continuous analysis method for planar multibody systems with joint clearance. Multibody System Dynamics, vol. 2, no. 1, p. 1-24, DOI:10.1023/A:1009759826529.
[45] Flores, P., Machado, M., Silva, M.T., Martins, J.M. (2011). On the continuous contact force models for soft materials in multibody dynamics. Multibody System Dynamics, vol. 25, no. 3, p. 357-375, D0l:10.1007/s11044-010-9237-4.

[46] Machado, M., Moreira, P., Flores, P., Lankarani, H.M. (2012). Compliant contact force models in multibody dynamics: Evolution of the Hertz contact theory. Mechanism and Machine Theory, vol. 53, p. 99-121, D0l:10.1016/j. mechmachtheory.2012.02.010.

[47] Lankarani, H., Nikravesh, P.E. (1990). A contact force model with hysteresis damping for impact analysis of multibody systems. Journal of Mechanical Design, vol. 112, no. 3, p. 369-376, D0l:10.1115/1.2912617.

[48] Bai, Z.F., Zhao, Y. (2013). A hybrid contact force model of revolute joint with clearance for planar mechanical systems. International Journal of Non-Linear Mechanics, vol. 48, p. 1536, D0I:10.1016/j.jjnonlinmec.2012.07.003.

[49] Suzuki, K. (2011). Artificial Neural Networks-Methodological Advances and Biomedical Applications. InTech publication, DOI:10.5772/644.

[50] MATLAB (2013). The MathWorks Inc, Natick.

[51] Lin, T., Horne, B.G., Tino, P., Giles, C.L. (1996). Learning longterm dependencies in NARX recurrent neural networks. IEEE Transactions on Neural Networks, vol. 7, no. 6, p. 1329-1338, DOI:10.1109/72.548162.

[52] Chan, R.W.K., Yuen, J.K.K., Lee, E.W.M., Arashpour, M. (2015). Application of nonlinear-autoregressive-exogenous model to predict the hysteretic behaviour of passive control systems. Engineering Structures, vol. 85, p. 1-10, D0l:10.1016/j. engstruct.2014.12.007.

[53] Chen, S., Bilings, S.A., Grant, P.M. (1990). Non-linear system identification using neural networks. International Journal of Control, vol. 51, no. 6, p. 1191-1214, DOI:10.1080/00207179008934126.

[54] Leontaritis, I.J., Bilings, S.A. (1985). Input-output parametric models for non-linear systems: Part I: Deterministic non-linear systems. International Journal of Control, vol. 41, no. 2, p. 303-328, DOI:10.1080/0020718508961129.

[55] Erkaya S. (2012). Analysis of the vibration characteristics of an experimental mechanical system using neural networks. Journal of Vibration and Control, vol. 18, no. 13, p. 20592072, DOI:10.1177/1077546311429059.

[56] Yildirim, Ş., Erkaya, S., Su Ş., Uzmay, İ. (2005). Design of neural networks model for transmission angle of a modified mechanism. Journal of Mechanical Science and Technology, vol. 19, no. 10, p.1875-1884, D0l:10.1007/BF02984266. 